\title{
The application of the "omental wrapping" technique with autologous onlay flap/graft ureteroplasty for the management of long ureteral strictures
}

\author{
Jie Wang ${ }^{1 \#}$, Baiyu Zhang ${ }^{2 \#}$, Jian Fan ${ }^{1 \#}$, Sida Cheng ${ }^{1}$, Shubo Fan ${ }^{1}$, Lu Yin ${ }^{1}$, Zhihua Li ${ }^{1}$, Hua Guan ${ }^{1}$, \\ Kunlin Yang ${ }^{1}$, Xuesong $\mathrm{Li}^{1}$ \\ ${ }^{1}$ Department of Urology, Peking University First Hospital, Institute of Urology, Peking University, National Urological Cancer Center, Beijing, \\ China; ${ }^{2}$ Department of Urology, The First Affiliated Hospital of Kunming Medical University, Kunming, China \\ Contributions: (I) Conception and design: J Wang, B Zhang; (II) Administrative support: X Li; (III) Provision of study materials or patients: X Li, \\ K Yang; (IV) Collection and assembly of data: S Fan, L Yin, Z Li, H Guan; (V) Data analysis and interpretation: J Fan, S Cheng; (VI) Manuscript \\ writing: All authors; (VII) Final approval of manuscript: All authors. \\ "These authors contributed equally to this work. \\ Correspondence to: Kunlin Yang; Xuesong Li. Department of Urology, Peking University First Hospital, Institute of Urology, Peking University, \\ National Urological Cancer Center, No. 8 Xishiku St, Xicheng District, Beijing 100034, China. Email: yangkunlin12345@163.com; \\ pineneedle@sina.com.
}

Background: To present our experience with the "omental wrapping" technique in laparoscopic and robotic ureteroplasty using onlay flaps or grafts for the management of long proximal or middle ureteral strictures.

Methods: This is a retrospective review of 25 patients with long proximal or middle ureteral strictures who underwent laparoscopic and robotic onlay flaps or grafts ureteroplasty using an omental flap to reinforce an anastomosis site between August 2018 and November 2019. Perioperative and follow-up data were collected. Results: Sixteen laparoscopic procedures and nine robotic procedures were performed successfully. Sixteen patients underwent ureteroplasty with lingual mucosal graft (LMG), and nine patients with appendiceal onlay flap (AOF). The median stricture length was $4 \mathrm{~cm}$ (range, $2-6 \mathrm{~cm}$ ). The mean operative time (OT) was $220.5 \pm 50.6 \mathrm{~min}$, the estimated blood loss (EBL) was $66.0 \pm 38.9 \mathrm{~mL}$, and the length of hospital stay (LHS) was 8.0 \pm 3.6 days. In the LMG group, four patients had tongue numbing and one had an oral ulcer, which relieved itself gradually without intervention. Two patients in the LMG group and four patients in the AOF group experienced urinary tract infection, and all responded well to antibiotic treatment. There were no complications attributed to "omental wrapping". The mean follow-up was $16.3 \pm 4.8$ months. According to the standards regarding improvement in clinical symptoms, relief of obstruction radiologically and a stable estimate glomerular filtration rate, our surgical success rate was $100 \%$.

Conclusions: The "omental wrapping" technique in laparoscopic and robotic onlay flaps or grafts ureteroplasty for long proximal or middle ureteral strictures is an efficient, safe, reproducible and simple technique.

Keywords: Ureteral strictures; omental wrapping; lingual mucosal graft (LMG); appendiceal flap

Submitted Apr 11, 2021. Accepted for publication May 25, 2021.

doi: 10.21037/tau-21-305

View this article at: https://dx.doi.org/10.21037/tau-21-305 


\section{Introduction}

Ureteral stricture is increasingly common but intractable because of its varied iatrogenic or non-iatrogenic aetiologies. As the disease progresses, the resulting urinary obstruction may cause hydronephrosis, infection, and irreversible renal function impairment if it is not immediately treated. Surgical interventions to reconstruct the urinary tract remain the definitive option with stable effects and include open surgery, laparoscopy and robotic techniques. Both conventional methods such as pyeloplasty, ureteroureterostomy and ureteral reimplantation and sophisticated methods such as ileal ureter replacement have been shown to relieve urinary tract obstruction and maximally preserving renal function $(1,2)$. The surgical treatment of choice should be based on a comprehensive consideration of the extent, position and nature of the ureteral stenosis. Furthermore, it is worth noting that laparoscopy and robotics are gradually replacing conventional open surgery in almost the whole field of reconstructive urology (3). Long ureteral strictures, especially in the middle and proximal segments, are difficult to repair, frequently demanding the performance of complicated reconstruction techniques. Both intestinal interposition and kidney autotransplantation are alternative techniques but with significant morbidity associated with intestinal substitution and vascular complications $(4,5)$. Ureteroplasty with oral mucosa grafts (buccal mucosa and lingual mucosa) and appendiceal onlay flaps (AOFs) are inspiring ways to repair narrow segments with less severe complications (6-8). A good blood supply and tension-free and watertight repair are the keys to the success of such operations.

Possessing multiple biological benefits including neovascularization, immune regulation, tissue healing and regeneration, the omentum is increasingly valuable in reconstructive surgical practice $(9,10)$. Early research reported the benefits of wrapping with omentum to provide vascularity in ureteral reconstruction (11-13). Herein, we describe the feasibility of "omental wrapping" following the onlay repair technique for ureteral strictures, including lingual mucosal grafts (LMGs) and AOF. We report our experience with the complications and effects of the "omental wrapping" technique with a small group of patients.

We present the following article in accordance with the STROBE reporting checklist (available at https://dx.doi. org/10.21037/tau-21-305).

\section{Methods}

Between August 2018 and November 2019, "omental wrapping" procedures were performed in laparoscopic and robotic ureteroplasty with LMG or AOF in 25 patients by the same experienced surgeon. The inclusion criterion was long proximal or middle ureteral strictures which unsuitable for conventional end-to-end anastomosis techniques, such as ureteroureterostomy and pyeloplasty. Patients were excluded if they had extensive ureteral strictures $(>6 \mathrm{~cm})$ or multifocal lesions and had undergone alternative reconstructive techniques, such as ileal ureteral replacement. Perioperative information of all patients was collected from our RECUTTER database (http://pkufh. yorktal.com/).

\section{Surgical technique}

The positioning and trocar placement were described in our previous reports $(14,15)$. After a LMG or an AOF was anastomosed to the ureteral stenosis, adjacent pedicled omentum that could be easily pulled and wrapped around the involved segment and that was both well vascularized and tension free was harvested. First, the prepared omentum flap was pulled up and passed posterior to the reconstructed site. Then, the marginal and medial edges were brought together anteriorly into a tube around the ureter and fixed with 3-0 absorbable suture. The omental flap was wrapped loosely without torsion. Finally, the lateral border of the "tube" was anastomosed with the peripheral peritoneum or connective tissues (Figure 1).

\section{Postoperative management and follow-up protocol}

The Foley catheters were removed within in one week. Patients were discharged with a double-J ureteral stent, and if placed, the nephrostomy tube was clamped. The double-J stents were removed 8-12 weeks postoperatively, and pyelography was performed. Follow-up was scheduled at 3, 6 and 12 months postoperatively and then annually. In addition to estimate glomerular filtration rate (eGFR) and renal ultrasound examination, imaging urodynamics examination (IUE), computed tomography urography (CTU), or cine magnetic resonance urography (cine MRU) were performed. Surgical success was defined as an improvement in clinical symptoms, relief of obstruction radiologically and a stable eGFR without serious complications. 

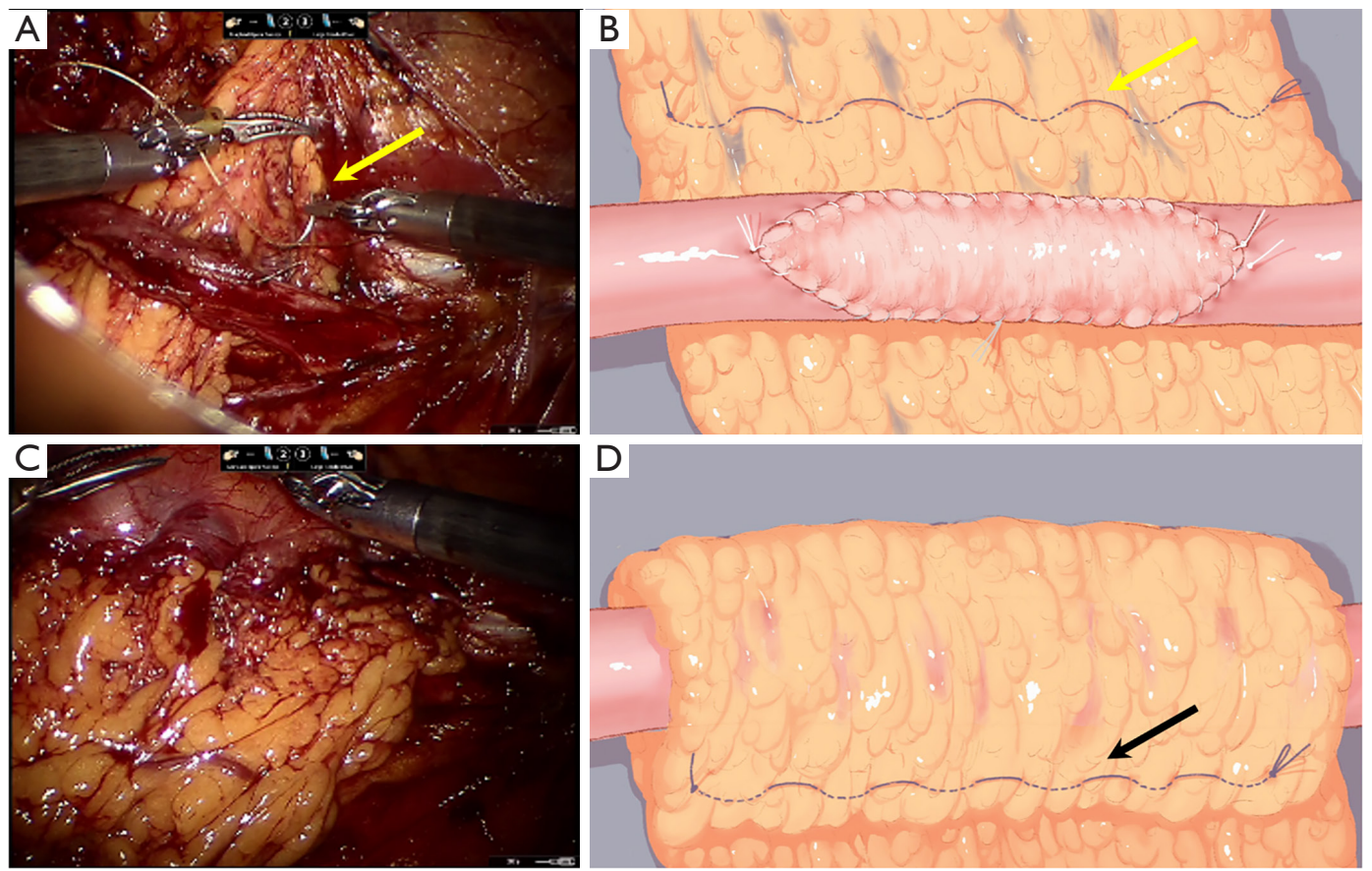

Figure 1 Intraoperative images and schematic diagrams of omentum wrapping technique. (A) After ureteroplasty completed, the omentum flap was lifted to the posterior side of the reconstructed site. Then the edge of the omentum was continuously sutured to the lateral peritoneum (yellow arrow); (B) the schematic diagram of (A), the edge of the omentum was continuously sutured to the lateral peritoneum which showed with a yellow arrow; (C) the ureteral anastomosis was completely wrapped by the omentum flap, and then the free end of omentum flap was continuously sutured and fixed to itself; (D) the schematic diagram of (C), the free end of omentum flap was continuously sutured and fixed to itself which showed with a black arrow.

\section{Statistical analysis}

Data were analyzed using the SPSS software version 22.0 (IBM Corporation, Armonk, NY, USA). Measurement data were expressed as mean \pm standard deviation or median (range), and enumeration data were expressed as number (percentage). Comparison between groups was performed using the Mann-Whitney $\mathrm{U}$ test and the Chi-square test. $\mathrm{P}$ value $<0.05$ were considered significant significance.

\section{Ethical statement}

The study was conducted in accordance with the Declaration of Helsinki (as revised in 2013). The study was approved by Ethics Committee of Peking University First Hospital (No.: 2019SR134) and individual consent for this retrospective analysis was waived.

\section{Results}

\section{Demographic and preoperative characteristics}

The demographic and preoperative characteristics of the overall population are summarized in Table 1 . The 25 patients were between 25 and 56 years old (mean age 39) and included 20 men and 5 women. The body mass index (BMI) was $25.1 \pm 3.4 \mathrm{~kg} / \mathrm{m}^{2}$ at the time of surgery. Iatrogenic injury remained the most common cause of ureteral strictures. The aetiology of ureteral stricture was idiopathic for 4 patients (16\%) and related to iatrogenic injuries for 21 patients (84\%), primarily holmium laser lithotripsy. Patients consistently had to endure multiple operations or long periods of stenting and nephrostomy to relieve discomfort and hydronephrosis. Eighteen (72\%) patients in our study had previously undergone failed surgeries, including ureteral stenting, balloon dilation, endoureterotomy, 
Table 1 Demographic and preoperative characteristics

\begin{tabular}{|c|c|c|c|}
\hline Variables & Overall & LMG & AOF \\
\hline \multicolumn{4}{|l|}{ Sex, n (\%) } \\
\hline Male & $20(80.0)$ & $13(81.2)$ & $7(77.8)$ \\
\hline Female & $5(20.0)$ & $3(18.8)$ & $2(22.2)$ \\
\hline $\mathrm{BMI}\left(\mathrm{kg} / \mathrm{m}^{2}\right)$, mean $\pm \mathrm{SD}$ & $25.1 \pm 3.4$ & $25.9 \pm 3.6$ & $23.6 \pm 2.3$ \\
\hline \multicolumn{4}{|l|}{ Stricture site, n (\%) } \\
\hline Proximal & $23(92.0)$ & $16(100.0)$ & $7(77.8)$ \\
\hline Middle & $2(8.0)$ & $0(0)$ & $2(22.2)$ \\
\hline \multicolumn{4}{|l|}{ Stricture aetiology, n (\%) } \\
\hline Ureterolithotomy & $5(20.0)$ & $3(18.8)$ & $2(22.2)$ \\
\hline Rectal cancer surgery & $1(4.0)$ & $1(6.2)$ & 0 \\
\hline UPJO & $4(16.0)$ & $4(25.0)$ & 0 \\
\hline Previous reconstruction, n (\%) & $18(72.0)$ & $12(75.0)$ & $6(66.7)$ \\
\hline Preoperative nephrostomy, n (\%) & $16(64.0)$ & $9(56.3)$ & $7(77.8)$ \\
\hline
\end{tabular}

BMI, body mass index; LMG, lingual mucosa graft; AOF, appendiceal onlay flap; UPJO, ureteropelvic junction obstruction.

Table 2 Perioperative characteristics and follow-up data

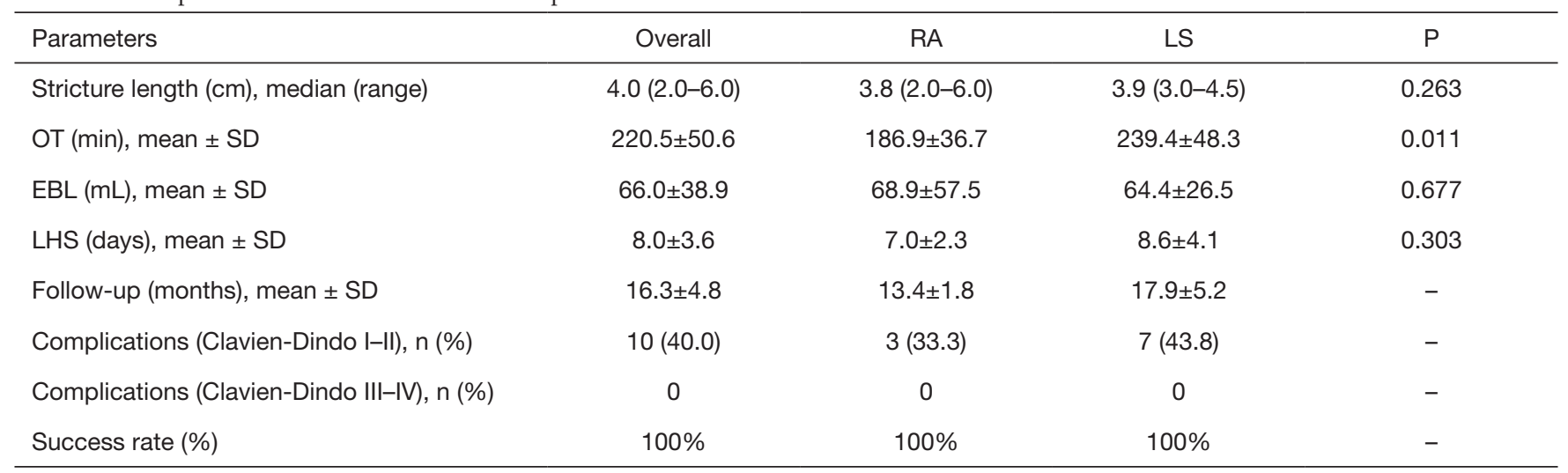

OT, operative time; EBL, estimated blood loss; LHS, length of hospital stays; RA, robot-assisted procedures; LS, laparoscopic procedures.

pyeloplasty and ureteroureterostomy, and fourteen patients (56\%) underwent more than one approach. Sixteen (64\%) patients required nephrostomy to relieve their symptoms and save affected kidney function.

\section{Perioperative and follow-up data}

The perioperative and follow-up data are summarized in Table 2. Sixteen laparoscopic procedures and 9 

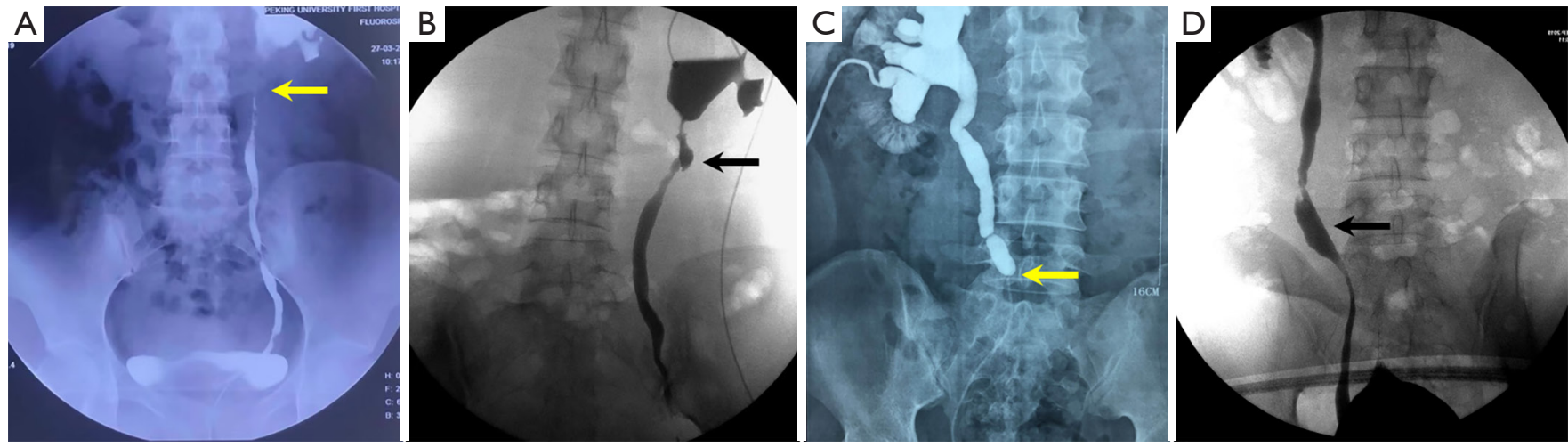

Figure 2 The comparison of patient's preoperative and postoperative imaging examination. (A) Preoperative anterograde and retrograde urography showing long proximal ureteral stricture of left side (yellow arrow); (B) IUE at 3 months after the LMG ureteroplasty showing an unobstructed ureter and a slightly dilated collection system, the LMG can be identified (black arrow); (C) preoperative anterograde and retrograde urography showing middle ureteral stricture of right side (yellow arrow); (D) IUE at 3 months after the AOF ureteroplasty showing an unobstructed ureter, the AOF can be identified (black arrow).

robotic procedures were performed successfully without intraoperative complications or open conversion. Sixteen patients underwent ureteroplasty with LMG, and nine underwent ureteroplasty with AOF. The median length of the strictures was $4 \mathrm{~cm}$ (range, 2-6 cm) according to intraoperative measurements. The mean operative time (OT) was $220.5 \pm 50.6 \mathrm{~min}$, the estimated blood loss (EBL) was $66.0 \pm 38.9 \mathrm{~mL}$, and the length of hospital stay (LHS) was 8.0 \pm 3.6 days. In the LMG group, four patients had tongue numbing and one had an oral ulcer, which relieved itself gradually without intervention. Two patients in the LMG group and four patients in the AOF group experienced urinary tract infection, and all responded well to antibiotic treatment. None of these were attributed to the "omental wrapping" technique.

At a mean follow-up of $16.3 \pm 4.8$ months, all procedures were deemed to be clinically and radiologically successful. All patients' double-J ureteral stents and nephrostomy tubes were removed successfully. Preoperative flank pain related to obstruction in seventeen patients was alleviated. Unobstructed ureter was confirmed by CTU, cine MRU or IUE (Figure 2) in 23 patients (92\%). Two patients in the LMG group experienced mild hydronephrosis without symptoms or deterioration of renal function during followup. Renal function improved or remained stable in all patients, with mean pre- and postoperative eGFR values of $84.2 \pm 19.5$ and $92.3 \pm 20.0 \mathrm{~mL} / \mathrm{min} / 1.73 \mathrm{~m}^{2}$, respectively. According to the standards regarding improvement in clinical symptoms, relief of obstruction radiologically and a stable eGFR, our surgical success rate was 100\%.

\section{Discussion}

Complex ureteral strictures, especially proximal and middle ureteral strictures, present a challenge to urologists. Extended tissue defects are a relative contraindication to endoscopic therapy or primary ureteroureterostomy due to their low success rate. Although intestinal interposition, such as ileal ureteral replacement, Yang-Monti ileal ureter and colon substitution for long and multiple ureteral strictures has been widely recognized, infection, mucus obstruction and metabolic disorders are troublesome complications that cannot be ignored (16). Due to the concerns with these existing procedures, the onlay repair technique has started to emerge and has already produced encouraging outcomes (6-8). Successful ureteral repair surgery relies on tension-free and watertight reconstruction with important principles, including minimization of ureteral manipulation and preservation of ureteral blood supply (17). One of the most attractive advantages of the onlay repair technique is that it makes full use of the ureteral stricture to become a "ureteral plate" without increasing tension. Robotic ureteroplasty with buccal mucosa grafts, is generally accepted with a high success rate of $83.3-90 \%(18,19)$. Li et al. reported their initial experience with laparoscopic LMG ureteroplasty with a 9-month follow-up and achieved success (6). In our experience, ureteroplasty with a LMG for repairing ureteral stricture has achieved excellent outcomes, 
but more cases and longer follow-up are needed (15). AOF are another practicable technique applied in onlay repair in a small group of patients with suitable ureteral strictures, with a reported success rate between 88.9-92\% $(14,20)$.

The main reasons for failure of ureteral reconstructive surgery using tissue transfer include ischaemia, infection and local adhesion. There is clinical concern regarding the lack of blood supply for the onlay flaps or grafts, especially the completely free oral mucosal graft. The omentum is a fold of the peritoneum connecting the stomach and other abdominal organs that provides coverage and protection and is composed of a trabecular connective tissue framework that carries blood vessels, lymphatic vessels, and fat pads. The omentum contains milky spots (MS), a network of capillaries, immune cells, and a mesothelial envelope that regulate immunity and metabolism (21). Multifaceted qualities, including revascularization, tissue regeneration, wound healing capacity and control of inflammation, make the omentum a valuable biological source in regenerative and reconstructive surgery $(9,22)$. One of the fundamental properties of the omentum is neovascularization in regional and adjacent structures to promote the healing process in ischaemic and inflamed tissue $(23,24)$. As early as 1997 , Teramachi et al. described "omental wrapping" as a useful supplementary method that reduced the occurrence of complications for intrathoracic tracheal reconstruction due to the speed of blood vessel ingrowth into the regenerated mucosa in animals (25). Yamashiro et al. reported that wrapping implanted artificial aortic grafts with omental flaps could prevent or reduce infection and that wellpreserved blood circulation could improve long-term outcomes (26). Dai et al. found that wrapping a pedicle omental flap around a oesophagogastric anastomosis site could decrease the incidence of anastomotic leakage and stricture, believing it worked partially by the pedicle omental flap forming new blood vessel networks (27). Shah et al. hypothesized that omental flaps around anastomoses after pancreaticoduodenectomy reduced the incidences of fistula and haemorrhage, perhaps benefitting from the flap providing a source of granulation tissue and neovascularization for prompt healing (28).

In urology, the omentum has been indicated to be an ideal tissue for fixation and repair according to the biological characteristics described above (29-31). In the treatment of retroperitoneal fibrosis, which obstructs and adheres to the ureter, "omental wrapping" has been used as a crucial protective manoeuvre after ureterolysis (32). In our experience, hyperplasia, adhesion of peripheral tissue and scarring around the ureteral stricture were commonly observed in patients who had undergone failed previous surgeries during our numerous reconstructive operations. These lesions, which intuitively impact ureteral peristalsis, exacerbate urinary obstruction by compression, sclerosis and even angulation of the ureter. However, "omental wrapping" is a potential and effective technique for controlling such risks to improve the surgical success rate. We performed laparoscopic and robotic ureteroplasty using LMG or AOF with "omental wrapping" for 25 patients who had long ureteral strictures. We believe that omental wrapping can prevent surgical anastomosis sites from ischaemia, infection and scar adhesions to a certain degree.

Limitations of this series include its retrospective nature and the small number of patients. A larger patient cohort and long-term evaluation are needed. This is a descriptive study and, as a result, we cannot prove a direct causal relationship between the omental wrapping technique and a high surgical success rate. Prospective and randomized studies are necessary to verify the value and rationality of this procedure.

\section{Conclusions}

As shown for the present 25 patients, the "omental wrapping" technique is reproducible and involves minimal costs over those of the base operation. We performed ureteroplasty using an onlay flap or graft with "omental wrapping" for all enrolled patients with an encouragingly high success rate. No serious complications or additional surgical burden was observed. In addition, in our experience, the "omental wrapping" technique did not add notably impact the total OT. The application of "omental wrapping" in laparoscopic and robotic ureteroplasty using onlay flaps or grafts for long proximal or middle ureteral strictures is efficient, safe, reproducible, and simple.

\section{Acknowledgments}

The authors are grateful for the entire staff of the Department of Urology, Peking University First Hospital. And acknowledge the excellent work of the medical illustrator Madam Xinying Ma, who drew figures for us. Funding: None.

\section{Footnote}

Reporting Checklist: The authors have completed the 
STROBE reporting checklist. Available at https://dx.doi. org/10.21037/tau-21-305

Data Sharing Statement: Available at https://dx.doi. org/10.21037/tau-21-305

Peer Review File: Available at https://dx.doi.org/10.21037/ tau-21-305

Conflicts of Interest: All authors have completed the ICMJE uniform disclosure form (available at https://dx.doi. org/10.21037/tau-21-305). XL serves as an unpaid editorial board member of Translational Andrology and Urology. The other authors have no conflicts of interest to declare.

Ethical Statement: The authors are accountable for all aspects of the work in ensuring that questions related to the accuracy or integrity of any part of the work are appropriately investigated and resolved. The study was conducted in accordance with the Declaration of Helsinki (as revised in 2013). The study was approved by Ethics Committee of Peking University First Hospital (No.: 2019SR134) and individual consent for this retrospective analysis was waived.

Open Access Statement: This is an Open Access article distributed in accordance with the Creative Commons Attribution-NonCommercial-NoDerivs 4.0 International License (CC BY-NC-ND 4.0), which permits the noncommercial replication and distribution of the article with the strict proviso that no changes or edits are made and the original work is properly cited (including links to both the formal publication through the relevant DOI and the license). See: https://creativecommons.org/licenses/by-nc-nd/4.0/.

\section{References}

1. Buffi NM, Lughezzani G, Hurle R, et al. Robot-assisted surgery for benign ureteral strictures: experience and outcomes from four tertiary care institutions. Eur Urol 2017;71:945-51.

2. Tracey AT, Eun DD, Stifelman MD, et al. Robotic-assisted laparoscopic repair of ureteral injury: an evidence-based review of techniques and outcomes. Minerva Urol Nefrol 2018;70:231-41.

3. Kapogiannis F, Spartalis E, Fasoulakis K, et al. Laparoscopic and Robotic Management of Ureteral Stricture in Adults. In Vivo 2020;34:965-72.
4. Xiong S, Zhu W, Li X, et al. Intestinal interposition for complex ureteral reconstruction: A comprehensive review. Int J Urol 2020;27:377-86.

5. Alameddine $M$, Moghadamyeghaneh Z, Yusufali A, et al. Kidney Autotransplantation: Between the Past and the Future. Curr Urol Rep 2018;19:7.

6. Li B, Xu Y, Hai B, et al. Laparoscopic onlay lingual mucosal graft ureteroplasty for proximal ureteral stricture: initial experience and 9-month follow-up. Int Urol Nephrol 2016;48:1275-9.

7. Zhao LC, Weinberg AC, Lee Z, et al. Robotic ureteral reconstruction using buccal mucosa grafts: a multiinstitutional experience. Eur Urol 2018;73:419-26.

8. Duty BD, Kreshover JE, Richstone L, et al. Review of appendiceal onlay flap in the management of complex ureteric strictures in six patients. BJU Int 2015;115:282-7.

9. Mazzaferro D, Song P, Massand S, et al. The omental free flap-a review of usage and physiology. J Reconstr Microsurg 2018;34:151-69.

10. Bass GA, Seamon MJ, Schwab CW. A Surgeon's History of the Omentum: From Omens To Patches To Immunity. J Trauma Acute Care Surg 2020;89:e161-e166.

11. Somerville JJ, Naude JH. Segmental ureteric replacement: an animal study using a free non-pedicled graft. Urol Res 1984;12:115-9.

12. Naude JH. Buccal mucosal grafts in the treatment of ureteric lesions. BJU Int 1999;83:751-4.

13. Atala A, Bauer SB, Soker S, et al. Tissue-engineered autologous bladders for patients needing cystoplasty. Lancet 2006;367:1241-6.

14. Wang J, Xiong S, Fan S, et al. Appendiceal onlay flap ureteroplasty for the treatment of complex ureteral strictures: initial experience of nine patients. J Endourol 2020;34:874-81.

15. Fan S, Yin L, Yang K, et al. Posteriorly augmented anastomotic ureteroplasty with lingual mucosal onlay grafts for long proximal ureteral strictures: 10 cases of experience. J Endourol 2021;35:192-9.

16. Kocot A, Kalogirou C, Vergho D, et al. Long-term results of ileal ureteric replacement: a 25 -year single-centre experience. BJU Int 2017;120:273-9.

17. Sun JY, Granieri MA, Zhao LC. Robotics and urologic reconstructive surgery. Transl Androl Urol 2018;7:545-57.

18. Lee Z, Waldorf BT, Cho EY, et al. Robotic ureteroplasty with buccal mucosa graft for the management of complex ureteral strictures. J Urol 2017;198:1430-5.

19. Lee Z, Lee M, Koster H, et al. A multi-institutional 
experience with robotic ureteroplasty with buccal mucosa graft: an updated analysis of intermediate-term outcomes. Urology 2021;147:306-10.

20. Jun MS, Stair S, Xu A, et al. A multi-institutional experience with robotic appendiceal ureteroplasty. Urology 2020;145:287-91.

21. Meza-Perez S, Randall TD. Immunological Functions of the Omentum. Trends Immunol 2017;38:526-36.

22. Shah S, Lowery E, Braun RK, et al. Cellular basis of tissue regeneration by omentum. PLoS One 2012;7:e38368.

23. García-Gómez I, Goldsmith HS, Angulo J, et al. Angiogenic capacity of human omental stem cells. Neurol Res 2005;27:807-11.

24. Di Nicola V. Omentum a powerful biological source in regenerative surgery. Regen Ther 2019;11:182-91.

25. Teramachi M, Okumura N, Nakamura T, et al. Intrathoracic tracheal reconstruction with a collagenconjugated prosthesis: evaluation of the efficacy of omental wrapping. J Thorac Cardiovasc Surg 1997;113:701-11.

26. Yamashiro S, Arakaki R, Kise Y, et al. Potential role of omental wrapping to prevent infection after treatment for infectious thoracic aortic aneurysms. Eur J Cardiothorac

Cite this article as: Wang J, Zhang B, Fan J, Cheng S, Fan S, Yin L, Li Z, Guan H, Yang K, Li X. The application of the "omental wrapping" technique with autologous onlay flap/graft ureteroplasty for the management of long ureteral strictures. Transl Androl Urol 2021;10(7):2871-2878. doi: 10.21037/tau-21305
Surg 2013;43:1177-82.

27. Dai JG, Zhang ZY, Min JX, et al. Wrapping of the omental pedicle flap around esophagogastric anastomosis after esophagectomy for esophageal cancer. Surgery 2011;149:404-10.

28. Shah OJ, Bangri SA, Singh M, et al. Omental flaps reduces complications after pancreaticoduodenectomy. Hepatobiliary Pancreat Dis Int 2015;14:313-9.

29. Ogunc G. Minilaparoscopic extraperitoneal tunneling with omentopexy: a new technique for CAPD catheter placement. Perit Dial Int 2005;25:551-5.

30. Dalela D, Gupta VP, Goel A, et al. Omental wrap around the renal pedicle: an adjunctive step to minimize morbidity and recurrence after lymphorenal disconnection for chyluria. BJU Int 2004;94:673-4.

31. Porzionato A, Sfriso MM, Macchi V, et al. Decellularized omentum as novel biologic scaffold for reconstructive surgery and regenerative medicine. Eur J Histochem 2013;57:e4.

32. Stein RJ, Patel NS, Quinn K, et al. Laparoscopic ureterolysis with omental wrap for idiopathic retroperitoneal fibrosis. BJU Int 2010;106:703-7. 